\title{
1 A geometric basis for surface habitat complexity and biodiversity
}

2 Damaris Torres-Pulliza ${ }^{1,2}$, Maria A. Dornelas ${ }^{3}$, Oscar Pizarro ${ }^{4}$, Michael Bewley ${ }^{4}$, Shane A.

3 Blowes $^{5,6}$, Nader Boutros $^{4}$, Viviana Brambilla ${ }^{3}$, Tory J. Chase ${ }^{7}$, Grace Frank ${ }^{7}$, Ariell Friedman ${ }^{4,8}$,

4 Mia O. Hoogenboom ${ }^{7}$, Stefan Williams ${ }^{4}$, Kyle J. A. Zawada ${ }^{3}$, Joshua S. Madin $^{1 *}$

5

$6{ }^{1}$ Hawai'i Institute of Marine Biology, University of Hawai'i, Kaneohe, HI, United States.

$7 \quad{ }^{2}$ Department of Biological Sciences, Macquarie University, Sydney, NSW, Australia.

$8{ }^{3}$ Centre for Biological Diversity, Scottish Oceans Institute, University of St Andrews, St Andrews

$9 \mathrm{KY16}$ 9TH, UK.

$10{ }^{4}$ Australian Centre for Field Robotics, University of Sydney, Sydney, NSW, Australia.

$11{ }^{5}$ German Centre for Integrative Biodiversity Research (iDiv) Halle-Jena-Leipzig, Deutscher Platz

12 5e, Leipzig 04103, Germany.

$13{ }^{6}$ Department of Computer Science, Martin Luther University Halle-Wittenberg, Am Kirchtor 1,

14 Halle (Salle) 06108, Germany.

$15{ }^{7}$ ARC Centre of Excellence for Coral Reef Studies and College of Science and Engineering, James

16 Cook University, Townsville, Queensland 4811, Australia.

$17{ }^{8}$ Greybits Engineering, Sydney, NSW, Australia.

$18 *$ Correspondence to: jmadin@hawaii.edu. 


\section{Abstract}

21 Structurally complex habitats tend to contain more species and higher total abundances than

22 simple habitats. This ecological paradigm is grounded in first principles: species richness scales

23 with area, and surface area and niche density increase with three-dimensional complexity. Here

24 we present a geometric basis for surface habitats that unifies ecosystems and spatial scales.

25 The theory is framed by fundamental geometric constraints among three structure

26 descriptors - surface height, rugosity and fractal dimension-and explains $98 \%$ of surface

27 variation in a structurally complex test system: coral reefs. We then show how coral

28 biodiversity metrics (species richness, total abundance and probability of interspecific

29 encounter) vary over the theoretical structure descriptor plane, demonstrating the value of the

30 theory for predicting the consequences of natural and human modifications of surface

31 structure.

\section{Main text}

34 Most habitats on the planet are surface habitats-from the abyssal trenches to the tops of

35 mountains, from coral reefs to the tundra. These habitats exhibit a broad range of structural

36 complexities, from relatively simple, planar surfaces to highly complex three-dimensional

37 structures. Currently, human and natural disturbances are changing the complexity of habitats

38 faster than at any time in history ${ }^{1-4}$. Therefore, understanding and predicting the effects of

39 habitat complexity changes on biodiversity is of paramount importance ${ }^{5}$. However, empirical

40 relationships between commonly-used descriptors of structural complexity and biodiversity are 
41 variable, often weak or contrary to expectation ${ }^{6-10}$. Moreover, there are no standards for

42 quantifying structural complexity, precluding general patterns in the relationship between

43 structure and diversity from being identified in different habitats. We therefore propose a new

44 geometric basis for surface habitats that integrates and standardises existing surface

45 descriptors ${ }^{8,10}$.

47 In theory, species richness scales with surface area according to a power law ${ }^{11}$. Island

48 biogeography theory articulates that this relationship arises out of extinction and colonization,

49 as larger areas provide larger targets for species to colonize and a greater variety of habitats

50 allowing species to coexist ${ }^{12}$. Our geometric theory builds on these ideas by exploring the

51 notion that habitat surfaces with the same rugosity (defined here as surface area per planar

52 area) can exhibit a range of different forms (Fig. 1). Total surface area is the integration of

53 component areas at the smallest scale (i.e., resolution), but it does not explain how these

54 component areas fold and fill the three-dimensional spaces they occupy. Rather, fractal

55 dimension quantifies space-filling at different scales ${ }^{13}$. Space-filling promotes species co-

56 existence by dividing surface area into a greater variety of structural elements ${ }^{14}$, microhabitats

57 and niches ${ }^{15}$ (e.g., high and low irradiance; small and large spaces; fast and slow flow). This

58 variety of niches allows species to coexist (e.g. different competitors, or predator and prey ${ }^{16}$ )

59 and therefore enhances biodiversity ${ }^{17,18}$. We posit that there is a fundamental geometric

60 constraint between surface rugosity and fractal dimension: for a given surface rugosity, an

61 increase in fractal dimension will result in a reduction of the surface's mean height (Fig. 1). As

62 the basis for a geometric theory, we mathematically derived the trade-off between surface 
63 rugosity $(R)$, fractal dimension $(D)$ and surface height range $(\Delta H)$ as (see Methods for

64 derivation):

65

$66 \frac{1}{2} \log \left(R^{2}-1\right)+\log \left(\frac{L}{L_{0}}\right)(3-D)=\log \left(\frac{\Delta H}{\sqrt{2} L_{0}}\right) \quad$ Eq. 1

67

68 Where $L$ is the surface linear extent and $L_{0}$ is the resolution (i.e., the smallest scale of

69 observation). $R$ and $D$ are both dimensionless, with $R \geq 1$ and $2 \leq D \leq 3 ; \Delta H$ is dimensionless

70 when standardised by resolution $L_{0}$, with $\frac{\Delta H}{\sqrt{2} L_{0}} \geq 0$. When rugosity is expressed as $R^{2}-1$ (with $R^{2}-1$

$71 \geq 0)$ and height range as $\frac{\Delta H}{\sqrt{2} L_{0}}$, Eq. 1 is a plane equation. Moreover, it is clear that any one of the

72 surface descriptors can easily be expressed in terms of the other two, highlighting that any of

73 the three variables is required, but not sufficient alone, to describe the structural complexity of

74 a surface habitat.

75

76 Results

77 To test the theory, we examined associations among surface rugosity, fractal dimension and

78 height range across coral reef habitat patches. Coral reefs are ideal ecosystems for testing a

79 theory of surface habitats, because they are structurally complex surface habitats constructed

80 in large part by the reef-building scleractinian corals that, in turn, live upon the habitat (i.e.,

81 corals are autogenic ecosystem engineers $\left.{ }^{19}\right)$. Structural complexity affects biodiversity in

82 general $^{20}$ and of coral reefs in particular ${ }^{21}$. Using Structure from Motion (SfM), we estimated

83 surface rugosity (expressed as the $\log$ of $R^{2}-1$ ), fractal dimension $(D)$ and height range (as the 
$84 \log$ of $\frac{\Delta H}{\sqrt{2} L_{0}}$ ) from digital elevation models (DEMs) for 591 reef patches of $4 \mathrm{~m}^{2}$ at 21 reef sites

85 encircling Lizard Island on the Great Barrier Reef, Australia (see Methods). Analyses of the

86 structure of these patches reveal that while rugosity, fractal dimension and surface height

87 range are not independent, they have substantial independent variation $\left(r^{2}\right.$ for pairwise

88 relationships between surface descriptors ranging between $3 \%$ and $30 \%$, Fig. 2a-c). However,

89 when framed together, the three descriptors formed a plane, whereupon the trivially measured

90 surface descriptors, rugosity and height range, captured $98 \%$ of the variation in $D$ (Fig. 2 d). The

91 remaining $2 \%$ of the variation occurs because real surfaces do not necessarily behave like

92 fractals (i.e., are self-similar) across a wide range of scales (Extended Data Fig. 5). The

93 observation that the structure of nearly all measured reef patches fell upon a plane delineated

94 by three simple surface descriptors highlights the fundamental geometric constraints of surface

95 habitats. If fractal dimension increases, then either rugosity increases, or height range

96 decreases, or both. All three descriptors are essential for capturing structural complexity

97 because they explain different elements of surface geometry: height range captures patch scale

98 variation, rugosity captures fine scale variation (which sums to surface area), and fractal

99 dimension captures degree of space filling when transitioning from broad to fine scales

100 (Extended Data Fig. 1a).

101

102 Different reef locations, with different ecological and environmental histories, occupied

103 different regions on the surface descriptor plane (Fig. 3). For example, one site that was

104 stripped of living coral during back-to-back tropical cyclones ${ }^{22}$ largely occupied the region of the

105 plane where rugosity, fractal dimension and surface height range are all low (Fig. 3a); that is, 
the patches at this site were closest to a theoretical flat surface. Another site also impacted by

107 the cyclones but left littered with dead coral branches, had similar levels of rugosity and height

108 range, but fractal dimension was relatively high (Fig. 3b). In contrast, a site containing several

109 large colonies of living branching coral had patches with the highest fractal dimension and

110 rugosity, yet the height range of these patches was low (Fig. 3c) reflecting the approximately

111 uniform height of living branching corals in shallow waters where water depth and tidal range

112 constrains colony growth. Meanwhile, a site containing large hemispherical Porites corals had

113 patches with large height ranges and high rugosity but lower fractal dimension (Fig. 3d). Three

114 sites contained patches with similar distributions of rugosities (Fig. 3b,d,f), and therefore similar

115 surface areas. However, these sites ranged from smooth reef surfaces with large holes (Fig. 3e)

116 to highly bumpy surfaces with no holes (Fig. 3b), demonstrating why rugosity alone does not

117 capture structural complexity and how varying mixtures of structural components dictate

118 habitat complexity ${ }^{14}$.

120 Finally, to connect the geometric variables to biodiversity, we examined how species richness,

121 total abundance and diversity (measured as the probability of interspecific encounter ${ }^{23}$ ) varied

122 across the surface descriptor plane. Strong ecological feedbacks occur between coral reef

123 habitat structure and coral biodiversity metrics. Coral reef structures are largely created by

124 corals, but their structure is mechanistically affected by environmental conditions such as tidal

125 range, currents, storm impacts and wave exposure. For instance, coral larvae are poor

126 swimmers and are more likely to settle in reef patches with small-scale complexity, because

127 they get entrapped by micro-eddies ${ }^{24}$. At the same time, more intricate coral structures (with 
128 higher fractal dimension, $D$ ) are more likely to be damaged or dislodged during storms that

129 flatten reef patches ${ }^{25,26}$. Species-area theory predicts that species richness and abundances

130 should be highest in patches with the greatest surface area ${ }^{11}$ (i.e., highest rugosity). We

131 predicted that higher fractal dimension would also enhance species richness and abundance,

132 because of niche diversity (i.e., increases in surface area at different scales), and that this effect

133 would be additional to overall surface area. The surface descriptor plane allows estimating the

134 combined effects of not just area, but also niche differentiation associated with fractal

135 dimension and height range $\mathrm{10,15}^{10,}$

137 We examined geometric-biodiversity coupling for a large plot, containing 261 of the $4 \mathrm{~m}^{2}$ reef

138 patches, in which 9,264 coral colonies of 171 species were recorded (see Methods). Contrary to

139 expectation, we found that all biodiversity metrics considered peaked in reef patches with

140 intermediate surface rugosities (Fig. 4a shows diversity, and Extended Data Table 2 includes

141 species richness and abundance). Indeed, several recent studies have argued that the

142 relationship should be unimodal because, as complexity increases, the amount of area available

143 for individuals to live declines ${ }^{27,28}$. However, biodiversity metrics also tended to increase

144 monotonically in association with patches with higher fractal dimension and smaller height

145 range (Extended Data Fig. 7). The explanatory power of reef geometry on biodiversity metrics

146 was over $50 \%$ (Extended Data Table 1) -5 to $45 \%$ higher than any surface descriptor alone.

147 Explaining this much variation in biodiversity is striking, given the number of other, non-

148 geometric processes that govern coral biodiversity, including environmental filtering, dispersal

149 and species interactions ${ }^{29}$. Because corals are autogenic ecosystem engineers, reciprocal 
150 causality is likely to strengthen and shape geometric-biodiversity coupling. For instance, high

151 rugosity is often generated by large hemispherical corals (e.g., Fig. 3d) that reduce the number

152 of individuals, and hence species, per area ${ }^{14}$. Subsequently, geometric-biodiversity coupling

153 may be weaker for other surface-associated taxa, such as fishes and invertebrates, and should

154 be tested. Nonetheless, our findings have implications for resilience following disturbances and

155 for restoration efforts that aim to maximise biodiversity ${ }^{30}$, specifically identifying the reef

156 structural characteristics that should be maintained (or built) to maximize biodiversity.

\section{Discussion}

A general, scale-independent geometric basis for surface habitats provides a much-needed way

to quantify habitat complexity across ecosystems and spatial scales. Meanwhile, creating three-

161 dimensional habitat surfaces is becoming increasingly accessible and cost effective, for example

162 using Structure from Motion ${ }^{31,32}$, both underwater and on land. The importance of surface

163 complexity as a determinant of habitat condition, biodiversity, and ecosystem function is well

164 recognised ${ }^{33}$, yet different metrics are typically used for different ecosystems, or different taxa

165 within the same ecosystem ${ }^{10}$. The general quantitative approach we propose is applicable

166 across surface habitats in both marine and terrestrial environments, allowing formal

167 comparisons examining whether geometric-biodiversity couplings differ among systems in

168 terms of both pattern and strength. The surface descriptor plane uncovered here clearly

169 defines the fundamental geometric constraints acting to shape surface habitats, and

170 consequently, how changes in surface geometry affect biodiversity. Nonetheless, there remain

171 several unknowns about the surface descriptor plane and its associations with biodiversity 
172 metrics that require further exploration. These unknowns range from technical limitations (e.g.,

173 how does the theory translate from digital elevation models that exclude overhanging surfaces

174 to 3D surface meshes?) to ecological patterns (e.g., how do different types of structural

175 components, such as different mixtures of branching and hemispherical corals or live and dead

176 elements ${ }^{14,34}$, mediate geometric-biodiversity coupling?).

178 As powerful ecosystem engineers, humans are modifying the planet through the structures we

179 destroy, both physically and indirectly via environmental change ${ }^{4}$, and those we construct.

180 Indeed, human-modified structures differ significantly in their geometry from nature-built

181 structures ${ }^{35}$. Determining how biodiversity, conservation status and recovery rates relate to

182 habitat complexity measures is paramount in the Anthropocene. The approach we propose

183 here allows for predictions of the biodiversity consequences of these structural changes across

184 land and seascapes.

186 Methods

187 Geometric theory for surface habitats. The variation method for calculating fractal dimension

$188 D$ measures the mean height range of a surface at different scales ${ }^{36,37}$. At the broadest scale,

189 the linear extent $L$, the surface height range is $\Delta H$ (Extended Data Fig. 1a). At the finest scale,

190 the resolution $L_{0}$, the height range $\left(\Delta H_{0}\right)$ is the mean of height ranges of all the component

191 areas at that scale. The slope $S$ of the resulting log-log relationship (shown in Extended Data Fig.

192 1a) is: 
$193 S=\frac{\log (\Delta H)-\log \left(\Delta H_{0}\right)}{\log (L)-\log \left(L_{0}\right)}$

Eq. 2

194 Where fractal dimension is ${ }^{37}$ :

$195 D=3-S$

Eq. 3

196 Rearranging Eq. 2 gives:

$197 S=\frac{\log \left(\Delta H / \Delta H_{0}\right)}{\log \left(L / L_{0}\right)}$

Eq. 4

198 Surface area $A$ can be estimated by summing areas $A_{0}$ at the finest grain $L_{0}$. Given the mean

199 height range $\Delta H_{0}$ at $L_{0}$, we assume any finer scale detail is not observable, and we calculate $A_{0}$

200 from the minimal surface consistent with $\Delta H_{0}$ (Extended Data Fig. 1b) as:

$201 A_{0}=\frac{L_{0}}{2} \sqrt{2 \Delta H_{0}^{2}+4 L_{0}^{2}}$

Eq. 5

202 And then multiply by the number of component areas $\left(\frac{L^{2}}{L_{0}^{2}}\right)$ giving:

$203 \quad A=\frac{L^{2}}{2 L_{0}} \sqrt{2 \Delta H_{0}^{2}+4 L_{0}^{2}}$

Eq. 6

204 Surface rugosity is ${ }^{32}$ :

$205 \quad R=\frac{A}{L^{2}}$

Eq. 7

206 Substituting $A$ for Eq. 6 and rearranging gives:

$207 \quad R=\sqrt{\frac{\Delta H_{0}^{2}}{2 L_{0}^{2}}+1}$

Eq. 8

208 Rearranging for $\Delta H_{0}$ gives:

$209 \Delta H_{0}=\sqrt{2} L_{0} \sqrt{R^{2}-1}$

Eq. 9

210 And substituting into Eq. 4 gives:

$211 S=\frac{\log \left(\frac{\Delta H}{\sqrt{2} L_{0} \sqrt{R^{2}-1}}\right)}{\log \left(\frac{L}{L_{0}}\right)}$

Eq. 10 
212 Leading to:

$213 D=3-\frac{\log \left(\frac{\Delta H}{\sqrt{2} L_{0} \sqrt{R^{2}-1}}\right)}{\log \left(\frac{L}{L_{0}}\right)}$

Eq. 11

214 Further rearranging gives a plane as Eq. 1 in the main text. The boundaries equations for the

215 limits of fractal dimension $D$ are:

$216 \Delta H_{D=2}=\sqrt{2} L \sqrt{R^{2}-1}$

Eq. 12

$217 \Delta H_{D=3}=\sqrt{2} L_{0} \sqrt{R^{2}-1}$

Eq. 13

Coral reef surface field study. Twenty-one reef flat sites were selected approximately $1 \mathrm{~km}$

221 spatial arrangement of the sites captured a broad range of habitats that were shaped

222 predominantly by wave exposure generated by prevailing southeast trade winds ${ }^{22}$. Mean water

223 depth across all study sites range between 2 to 3.5 meters. In 2014, at the Trimodal site, we

224 used an Iver2 Autonomous Underwater Vehicle ${ }^{38}$ to collect 45,000 georeferenced overlapping,

225 stereo-pair images of an approximately $30 \mathrm{~m}$ by $50 \mathrm{~m}$ section of the reef crest (Fig. 4c). In 2016,

226 at all 21 sites, we used the spiral method ${ }^{39}$, which involves swimming a camera rig that

227 unspools from a central point to capture approximately 3000 overlapping, stereo-pair images of

228 approximately $130 \mathrm{~m}^{2}$ of reef crest (Extended Data Fig. 3). We used a simultaneous localisation

229 and mapping approach ${ }^{40}$ fusing GPS, stereo imagery and altitude information to provide an

230 initial pose estimate for the cameras. We used Agisoft Metashape software to process the

231 images and produce a 3D dense cloud from which we derived a gridded digital elevation model

232 (DEM) and orthographic mosaic for coral annotation per site. The output resolution of all DEMs 
233 was $0.002 \mathrm{~m}$. We used DEMs in order to exclude overhanging surfaces (i.e., only one height for

234 each xy combination), because the degree to which overhangs are captured from plan view

235 photographic surveys is biased by the changing lighting conditions of the environment ${ }^{41}$ (e.g.,

236 the sun angle, cloud cover, water turbidity, etc.). On the other hand, plan view surveys were

237 preferred in order to reduce the time costs associated with capturing stereo pairs from multiple

238 view angles over large areas. The use of DEMs will underestimate surface rugosity and fractal

239 dimension; i.e., the reason why $D$ tended to range below 2.6. However, given that overhanging

240 structures were rare at our study sites, $R$ and $D$ measures are likely to exhibit the correct rank

241 order for patches.

243 Given the lack of coral cover following the 2016 mass bleaching event on the $\mathrm{GBR}^{22}$, we used

244 the 2014 Trimodal large plot to quantify geometric-biodiversity relationships (Extended Data

245 Fig. 2). The plot was divided into a contiguous grid of 2 by $2 \mathrm{~m}$ reef patches (Fig. 4c, black

246 squares). Patches of the orthographic mosaic were printed on underwater paper and used as

247 reference maps for in situ identification of all coral colonies of diameter $>5 \mathrm{~cm}$ to species by a

248 team of six researchers over four weeks. We focused on the reef crest and flat (shallower areas

249 in Fig. 4c) but also included reef edge and deeper reef. Colonies of unknown or hard to identify

250 species were photographed and identified in consultation with guide books and other

251 observers. Hemispherical Porites colonies were identified to genus due to the difficulty

252 differentiating among the few known species without collecting samples for microscopy. Colony

253 annotations were digitized over the orthographic mosaic using QGIS software (e.g., Fig. 4c,

254 white points). Only scleractinian corals were included for analyses. In total, 9,264 coral colonies 
of 171 species were observed within the 255 reef patches censused. Diversity was calculated as the probability of interspecific encounter (PIE), or 1 - Simpson diversity ${ }^{23}$.

258 Each of the circular DEMs had a central point, from which an 8 by $8 \mathrm{~m}$ square was centred

259 (Extended Data Fig. 3) and divided into 16 contiguous reef patches of 2 by $2 \mathrm{~m}$. DEMs for each 2

266 (i.e., $0.03125 \mathrm{~m}$ ). The height range within each grid at each scale were calculated, and then

267 averaged for that scale to avoid weighting the many estimates at smaller scales more than the

268 fewer estimates at larger scales when calculating the slope $S$. S was calculated for each patch by

269 fitting a linear model to the log of scale (i.e., grid sizes) versus the log of mean height. $D$ was

270 then calculated according to Eq. 3. $R$ was calculated according to Eq. 8. There are many ways to

271 estimate the surface area of a DEM, so we compared surface rugosity calculated from theory

272 (Eq. 8) with estimates based on surface area calculations using the surfaceArea function in the

273 package $s p^{43}$. The theory underestimated surface rugosity by approximately $5 \%$ (Extended Data

274 Fig. 4), because of the minimal area assumption (Extended Data Fig. 1b), but this disparity was

275 consistent across the range of rugosities. 
277 Analyses. Surface rugosity (expressed as $R^{2}-1$ ) and standardised height range (expressed as

$278 \frac{\Delta H}{\sqrt{2} L_{0}}$ ) were log-transformed (base 10) as per the plane equation (Eq. 1). Species richness and

279 abundance were sqrt-transformed, and diversity arcsin-transformed, for all analyses to improve

280 model residuals. Coefficients of determination ( $r$ square) of pairwise associations of the three

281 geometric variables were estimated by squaring Pearson correlation coefficients. Reef surfaces

282 were not perfectly fractal: mean height ranges at $L$ and $L_{0}$ anchor the theory (Extended Data Fig.

283 1a), but mean height ranges at scales intermediate to $L$ and $L_{0}$ could shift the overall

284 relationship, albeit subtlety. Therefore, we calculated the $r$ square for the surface descriptor

285 plane based on the deviances of empirically derived $D$ from theory derived $D$ (Extended Data

286 Fig. 5) (i.e., by dividing the residual sums of squares by the total sums of squares, and then

287 subtracting this value from one).

289 We quantified geometric-biodiversity relationships for the large plot at the Trimodal site using

290 both generalised additive models (GAMs) and linear models (LMs). We applied the default

291 smoother term to each surface descriptor for the GAMs and second-order polynomials for the

292 LMs, to allow for non-linear relationships among predictor and response variables. We

293 quantified the effect of each geometric variable separately on species richness, total abundance

294 and diversity (PIE), and then all together to assess improvement in explained variation as

295 adjusted r square values (Extended Data Fig. 7; Extended Data Table 1). We included the three

296 reef patches with no living coral, but also confirmed that removing these points had no

297 discernible influence on the geometric-biodiversity relationships. We also ran analyses

298 following the removal of the 5-6 highest rugosity patches that appeared to be largely 
responsible for producing the hump-shaped rugosity-biodiversity relationships (red curves,

301 reference degrees of freedom much greater than one for all biodiversity metrics, suggesting

302 significant non-linear effects for these surface descriptors ${ }^{44}$. Fractal dimension showed a linear

303 effect for richness and diversity, and so the smoother term was removed for these analyses.

304 Residuals for all models were approximately normal and were homogeneous when plotted

305 against predictor variables. The linear models with second-order polynomial terms gave the

306 same overall results as GAMs (Extended Data Fig. 7). That is, the polynomial term was

307 significant for the same terms that retained the smoother function in the GAMs. However, the

308 LMs had lower adjusted $r$ square values and so we presented the final results using GAMs (Fig.

309 4a; Extended Data Table 2).

311 All analyses, including model selection and diagnostics and figure creation, were conducted in

312 the statistical program language $\mathrm{R}^{45}$ and can be downloaded or cloned at GitHub

313 (https://github.com/jmadin/surface geometry).

315 Data and code availability

316 Source data and code for data preparation, statistical analyses and figures are available at

317 https://github.com/imadin/surface geometry. 
1. S. L. Pimm, C. N. Jenkins, R. Abell, T. M. Brooks, J. L. Gittleman, L. N. Joppa, P.H. Raven, C. M. Roberts, J. O. Sexton, The biodiversity of species and their rates of extinction, distribution, and protection. Science 344, 1246752 (2014).

2. T. Newbold, L. N. Hudson, S. L. L. Hill, S. Contu, I. Lysenko, R. A. Senior, L. Börger, D. J. of land use on local terrestrial biodiversity. Nature 520, 45-50 (2015).

3. Alvarez-Filip, L., Dulvy, N. K., Gill, J. A., Côté, I. M. \& Watkinson, A. R. Flattening of Caribbean

4. Millennium Ecosystem Assessment. 2005. Ecosystems and human well-being: biodiversity synthesis. World Resources Institute, Washington, D.C., USA. http://www.unep.org/maweb/en/index.aspx and the distribution of arthropod body lengths. Nature 314, 731-733 (1985). 
8. E. D. McCoy, S. S. Bell, Habitat structure: The evolution and diversification of a complex topic. in Habitat Structure: The physical arrangement of objects in space (eds. Bell S.S., McCoy E.D. \& Mushinsky H.R.) 3 - 27 (Springer, 1991).

9. M. W. Beck, Separating the elements of habitat structure: independent effects of habitat complexity and structural components on rocky intertidal gastropods. J. Exp. Mar. Biol. Ecol. 249, 29-49 (2000).

10. K. E. Kovalenko, S. M. Thomaz, D. M. Warfe, Habitat complexity: approaches and future directions. Hydrobiologia 685, 1-17 (2012).

11. O. Arrhenius, Species and Area. J. Ecol. 9, 95-99 (1921).

12. R. H. MacArthur, E. O. Wilson, The Theory of Island Biogeography (Princeton Univ. Press, 1967).

13. B. B. Mandelbrot, The Fractal Geometry of Nature (W. H. Freeman and Co., 1983).

14. Tokeshi, M. \& Arakaki, S. Habitat complexity in aquatic systems: fractals and beyond. Hydrobiologia 685, 27-47 (2012).

15. M. P. Johnson, N. J. Frost, M. W. J. Mosley, M. F. Roberts, S. J. Hawkins, The areaindependent effects of habitat complexity on biodiversity vary between regions. Ecol. Lett.

16. P. Chesson, Mechanisms of maintenance of species diversity. Annu. Rev. Ecol. Syst. 31, 343366 (2000).

17. E. R. Pianka, Evolutionary Ecology (Harper and Row, 1988). (1990). 
19. C. G. Jones, J. H. Lawton, M. Shachak, Positive and negative effects of organisms as physical ecosystem engineers. Ecology 78, 1946-1957 (1997).

364 20. J. H. Brown, V. K. Gupta, B. L. Li, B. T. Milne, C. Restrepo, G. B. West, The fractal nature of nature: power laws, ecological complexity and biodiversity. Philos. T. R. Soc. B. 357, 619626 (2002).

367 21. N. A. J. Graham, K. L. Nash, The importance of structural complexity in coral reef ecosystems. Coral Reefs 32, 315-326 (2012).

22. J. S. Madin, A. H. Baird, T. C. L. Bridge, S. R. Connolly, K. J. A. Zawada, M. Dornelas,

370 Cumulative effects of cyclones and bleaching on coral cover and species richness at Lizard Island. Mar. Ecol. Prog. Ser. 604, 263-268 (2018).

23. Hurlbert, S. H. The Nonconcept of Species Diversity: A Critique and Alternative Parameters. Ecology 52, 577-586 (1971).

374 24. T. Hata, J. S. Madin, V. R. Cumbo, M. Denny, J. Figueiredo, S. Harii, C. J. Thomas, A. H. Baird, 375 Coral larvae are poor swimmers and require fine-scale reef structure to settle. Sci. Rep. 7, $376 \quad 2249$ (2017).

377 25. J. S. Madin, S. R. Connolly, Ecological consequences of major hydrodynamic disturbances on $378 \quad$ coral reefs. Nature 444, 477-480 (2006).

379 26. L. Alvarez-Filip, J. A. Gill, N. K. Dulvy, A. L. Perry, A. R. Watkinson, I. M. Côté, Drivers of 380 region-wide declines in architectural complexity on Caribbean reefs. Coral Reefs 30, 1051$381 \quad 1060$ (2011). 
382

383

384

385

386

387

388

389

390

391

392

393

394

395

396

397

398

399

400

401

402

27. Allouche, O., Kalyuzhny, M., Moreno-Rueda, G., Pizarro, M. \& Kadmon, R. Areaheterogeneity tradeoff and the diversity of ecological communities. Proc. Nat. Acad. Sci. 109, 17495-17500 (2012).

28. Paxton, A. B., Pickering, E. A., Adler, A. M., Taylor, J. C. \& Peterson, C. H. Flat and complex temperate reefs provide similar support for fish: Evidence for a unimodal species-habitat relationship. PLoS ONE 12, e0183906 (2017).

29. Huston, M. A. Patterns of species diversity on coral reefs. Ann. Rev. Ecol. Syst. 16, 149-177 (1985).

30. Loke, L. H. L., Ladle, R. J., Bouma, T. J. \& Todd, P. A. Creating complex habitats for restoration and reconciliation. Ecological Engineering 77, 307-313 (2015).

31. Young, G. C., Dey, S., Rogers, A. D. \& Exton, D. Cost and time-effective method for multiscale measures of rugosity, fractal dimension, and vector dispersion from coral reef 3D models. PLoS ONE 12, e0175341 (2017).

32. A. Friedman, O. Pizarro, S. B. Williams, M. Johnson-Roberson, Multi-scale measures of rugosity, slope and aspect from benthic stereo image reconstructions. PLoS ONE 7, e50440 (2012).

33. E. Weiher, P. Keddy, Ecological Assembly Rules: Perspectives, Advances, Retreats. (Cambridge Univ. Press, 2004).

34. Bartholomew, A., Diaz, R. \& Cicchetti, G. New dimensionless indices of structural habitat complexity: predicted and actual effects on a predator's foraging success. Mar. Ecol. Prog. Ser. 206, 45-58 (2000). 
35. Strain, E. M. A. et al. Eco-engineering urban infrastructure for marine and coastal biodiversity: Which interventions have the greatest ecological benefit? Journal of Applied Ecology 55, 426-441 (2018).

36. B. Dubuc, S. W. Zucker, C. Tricot, J. F. Quiniou, D. Wehbi, Evaluating the fractal dimension of surfaces. P. Roy. Soc. A-Math. Phy. 425, 113-127 (1989).

37. G. Zhou, N. S-N. Lam, A comparison of fractal dimension estimators based on multiple surface generation algorithms. Comput. Geosci. 31, 1260-1269 (2005).

38. M. Johnson-Roberson, M. Bryson, A. Friedman, O. Pizarro, G. Troni, P. Ozog, J. C. Henderson, High-resolution underwater robotic vision-based mapping and threedimensional reconstruction for archaeology. J. Field Robot. 34, 625-643 (2017).

39. O. Pizarro, A. Friedman, M. Bryson, S. B. Williams, J. Madin, A simple, fast, and repeatable survey method for underwater visual 3D benthic mapping and monitoring. Ecol. Evol. 7,

40. I. Mahon, S. B. Williams, O. Pizarro, M. Johnson-Roberson, Efficient view-based SLAM using visual loop closures. IEEE T. Robot. 24, 1002-1014 (2008). to measure marine habitat structural complexity. Ecol. Evol. 7, 5669-5681 (2017). Lidar-Derived Bathymetry. J. Coastal Res. 10053, 6-15 (2009).

423 43. R. S. Bivand, E. Pebesma, V. Gómez-Rubio, Applied Spatial Data Analysis with R. Use R!, 2 ed $424 \quad$ (Springer, 2013). 
425 44. S. N. Wood, N. Pya, B. Saefken, Smoothing parameter and model selection for general

426 smooth models (with discussion). J. Am. Stat. Assoc. 111, 1548-1575 (2016).

427 45. R Core Team. R: A language and environment for statistical computing. R Foundation for

428 Statistical Computing, (Vienna, Austria 2019). https://www.R-project.org/.

\section{Acknowledgments}

431 We thank Dr. Anne Hoggett and Dr. Lyle Vail of the Lizard Island Research Station for their

432 support.

\section{Funding}

435 This work was supported by an Australian Research Council Future Fellowship (JM), the John

436 Templeton Foundation (MD, JM), a Royal Society research grant and a Leverhulme fellowship

437 (MD), an International Macquarie University Research Excellence Scholarship (DTP), two Ian

438 Potter Doctoral Fellowships at Lizard Island (DTP and VB), and an Australian Endeavour

439 Scholarship (TC).

\section{Author contributions}

442 The study was conceptualized by JSM, DTP, MD and OP. All authors collected the data. JSM and

443 OP developed the theory and JSM ran the analyses. JSM, DTP and OP developed the software

444 pipeline for data and produced the visualizations. The investigation was led by JSM, DTP, MD

445 and OP. JSM and MD led and funded the broader project, with additional field robotics 
resources provided by OP and SW. JSM wrote the first draft of the paper and all authors

447 reviewed at least one draft.

449 Competing interests

450 Authors declare no competing interests.

\section{Figure captions}

\section{Fig. 1 | Increasing fractal dimension (i.e., space filling) while keeping surface rugosity}

454 constant results in a decline in a surface's mean height range. A two-dimensional

455 representation of three hypothetical surface habitats with the same surface rugosities (a, b and

456 c). That is, the lengths of the lines $\mathbf{a}, \mathbf{b}$ and $\mathbf{c}$ are the same and occur over the same planar

457 extent (black points). However, line a fills less of its two-dimensional space (black rectangle)

458 than does line $\mathbf{c}$, and therefore has a lower fractal dimension.

Fig. 2 | Comparison of the geometric theory with field data. (a-c) Pairwise relationships

462 rugosity (as $R^{2}-1$ ); fractal dimension $D$; and surface height range (as $\frac{\Delta H}{\sqrt{2} L_{0}}$ ). Coefficients of

463 determination $\left(r^{2}\right)$ show the variance explained in the $y$-axis variable by the $x$-axis variable. (d)

464 When combined the three descriptors explain more than $98 \%$ of the variation in fractal

465 dimension $D$ despite reef surfaces not being perfectly fractal (see Methods). Field data are

466 points, and the surface descriptor plane is coloured by fractal dimension. 
468 Fig. 3 | The geometric diversity of coral reef habitats. Reef patches $(n=16)$ from a subset of six

469 sites are superimposed onto a two-dimensional representation of the surface descriptor plane

470 (colour used here to delineate sites). (a) North Reef; (b) Osprey; (c) Lagoon-2; (d) Resort; (e)

471 South Island; and (f) Horseshoe. Patch height range is greater in the top left corner and

472 decreases towards the bottom right corner. The corresponding DEMs and orthographic mosaics

473 show selected patches at each site to help visualise geometric differences.

475 Fig. 4 |Geometric-biodiversity coupling of coral reef habitats. (a) Predicted coral species

476 diversity (represented as probability of interspecific encounter) when plotted upon the surface

477 descriptor plane given by rugosity and fractal dimension (height range is greater in the top left

478 and decreases towards the bottom right, as per Eq. 1). Prediction contours are from the general

479 additive model summarised in Extended Data Table 2. (b) A digital elevation model of the large

480 plot with $n=255$ contiguous $2 \times 2$ m reef patches (black squares) capturing 9,264 coral colony

481 annotations (white points) representing 171 species. 


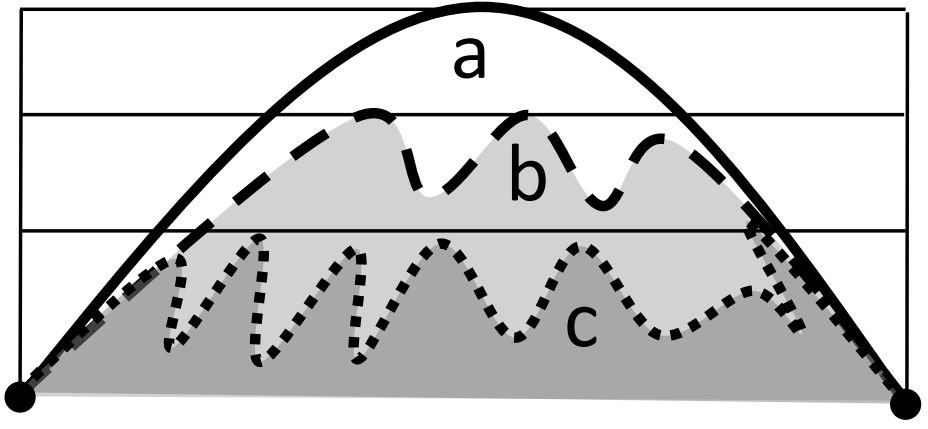


a
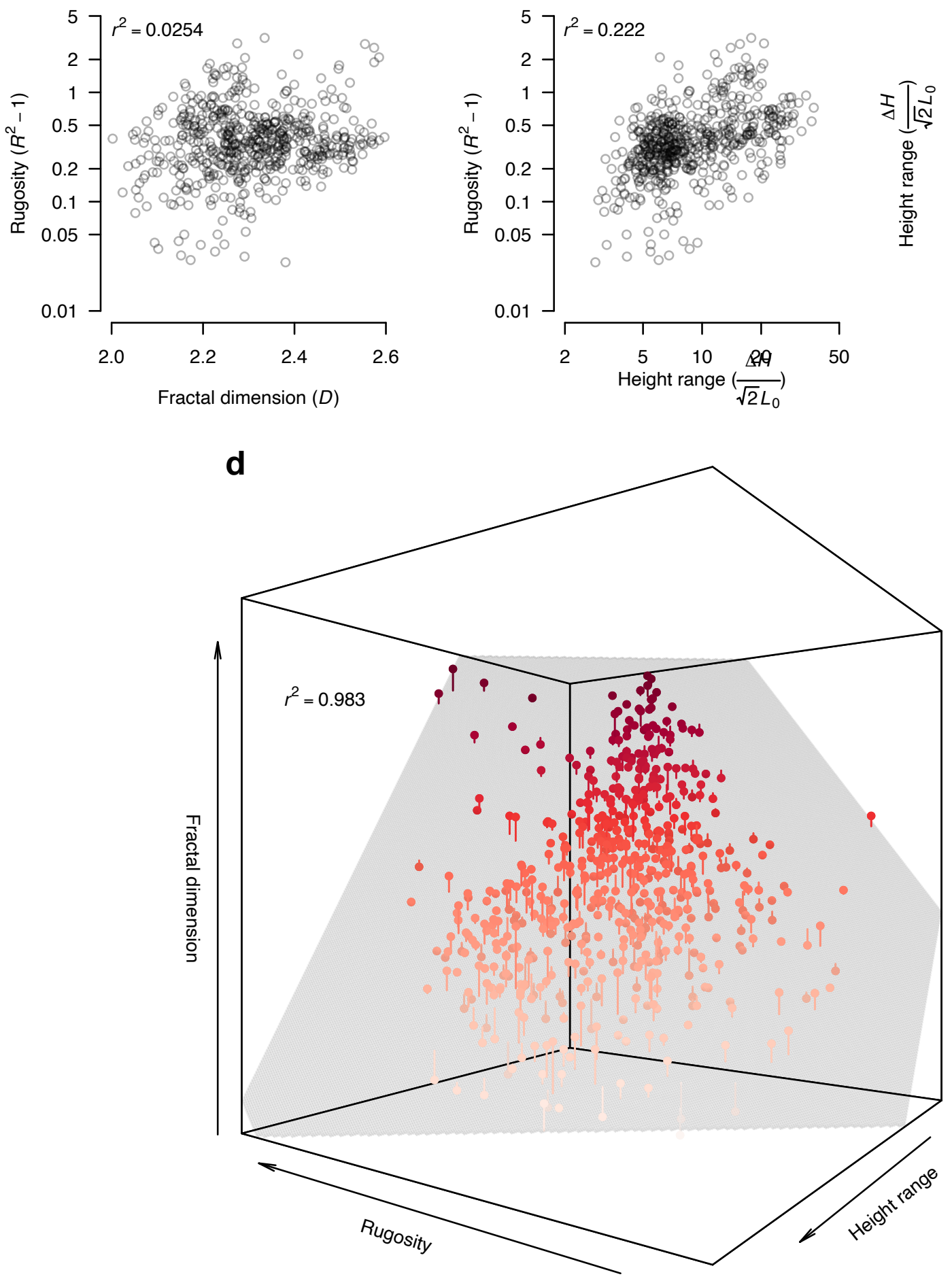
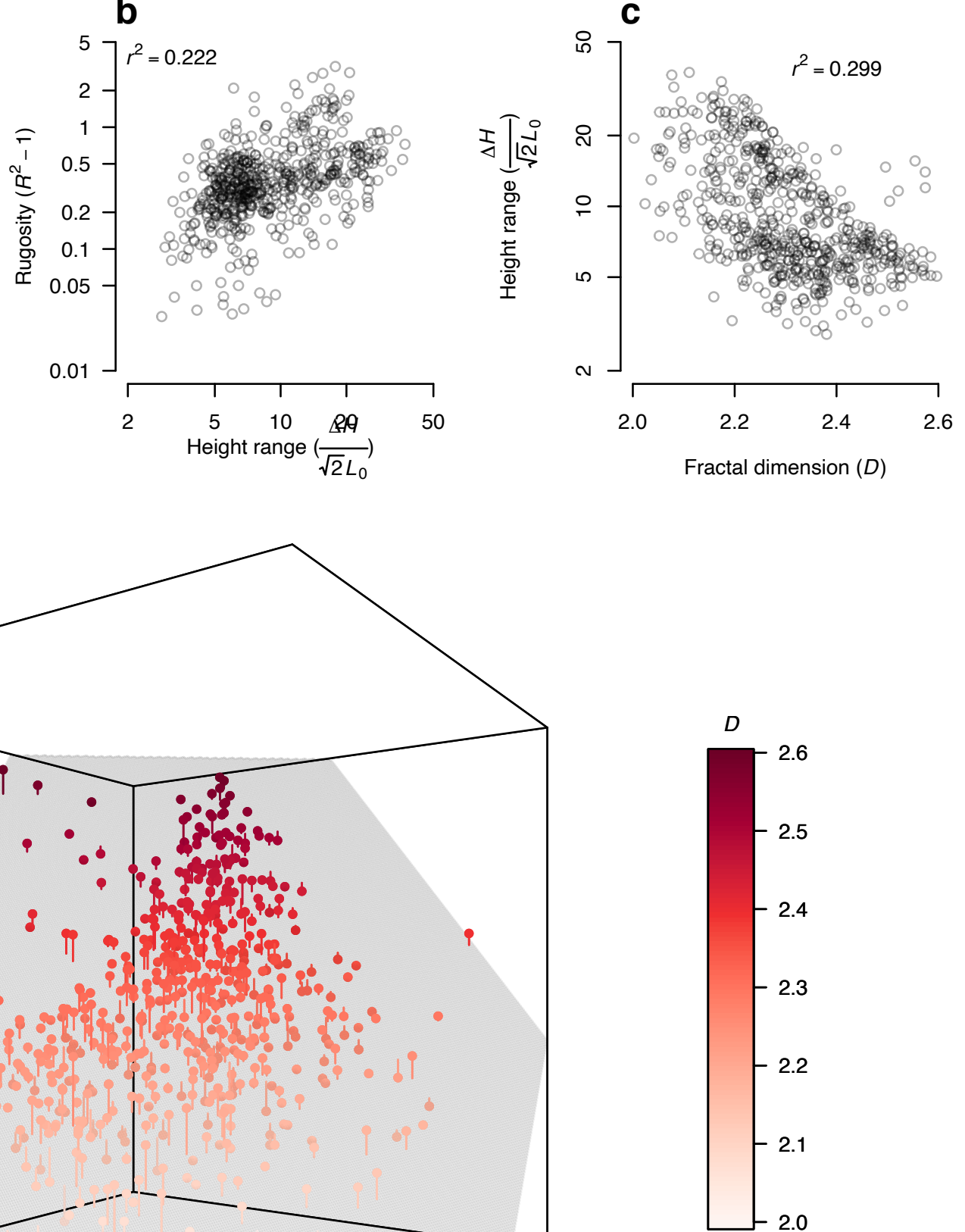


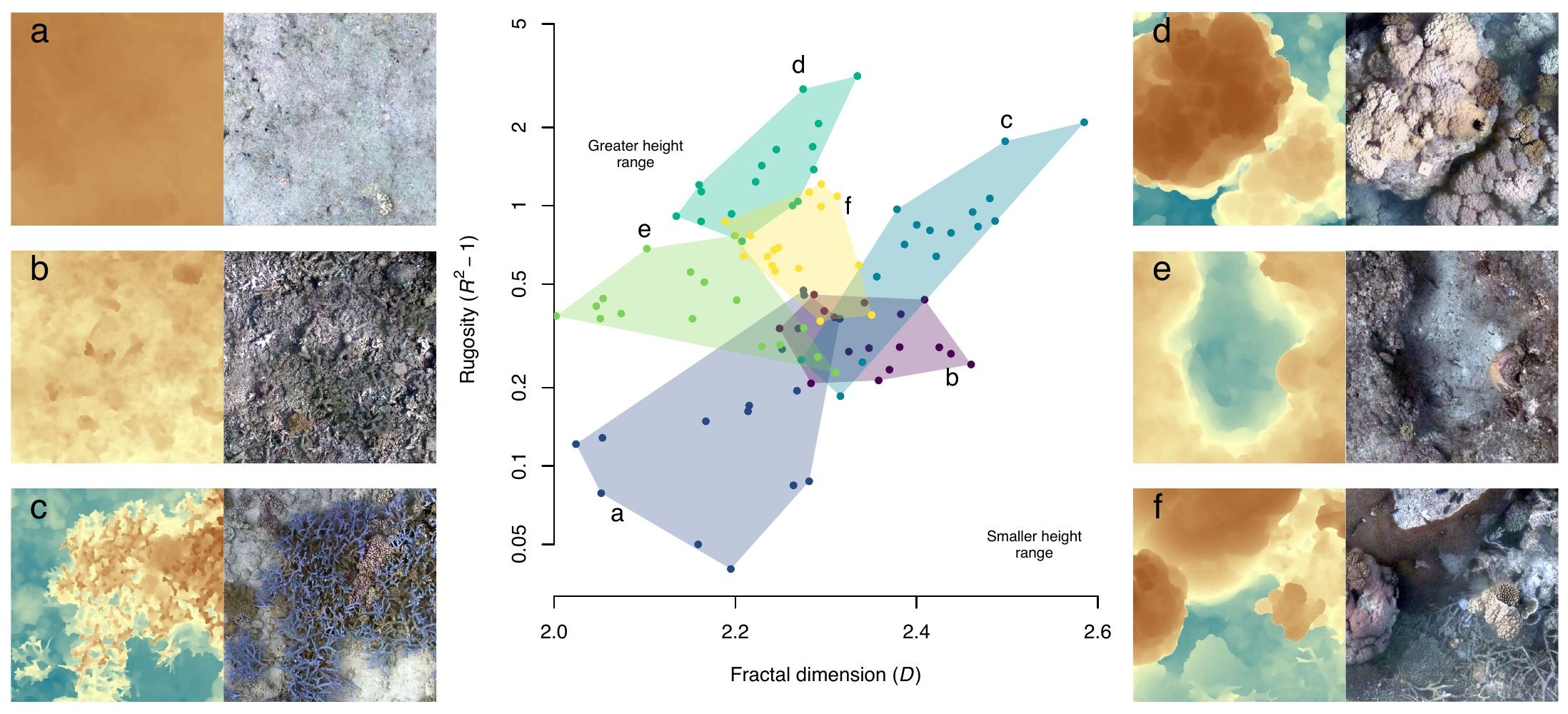



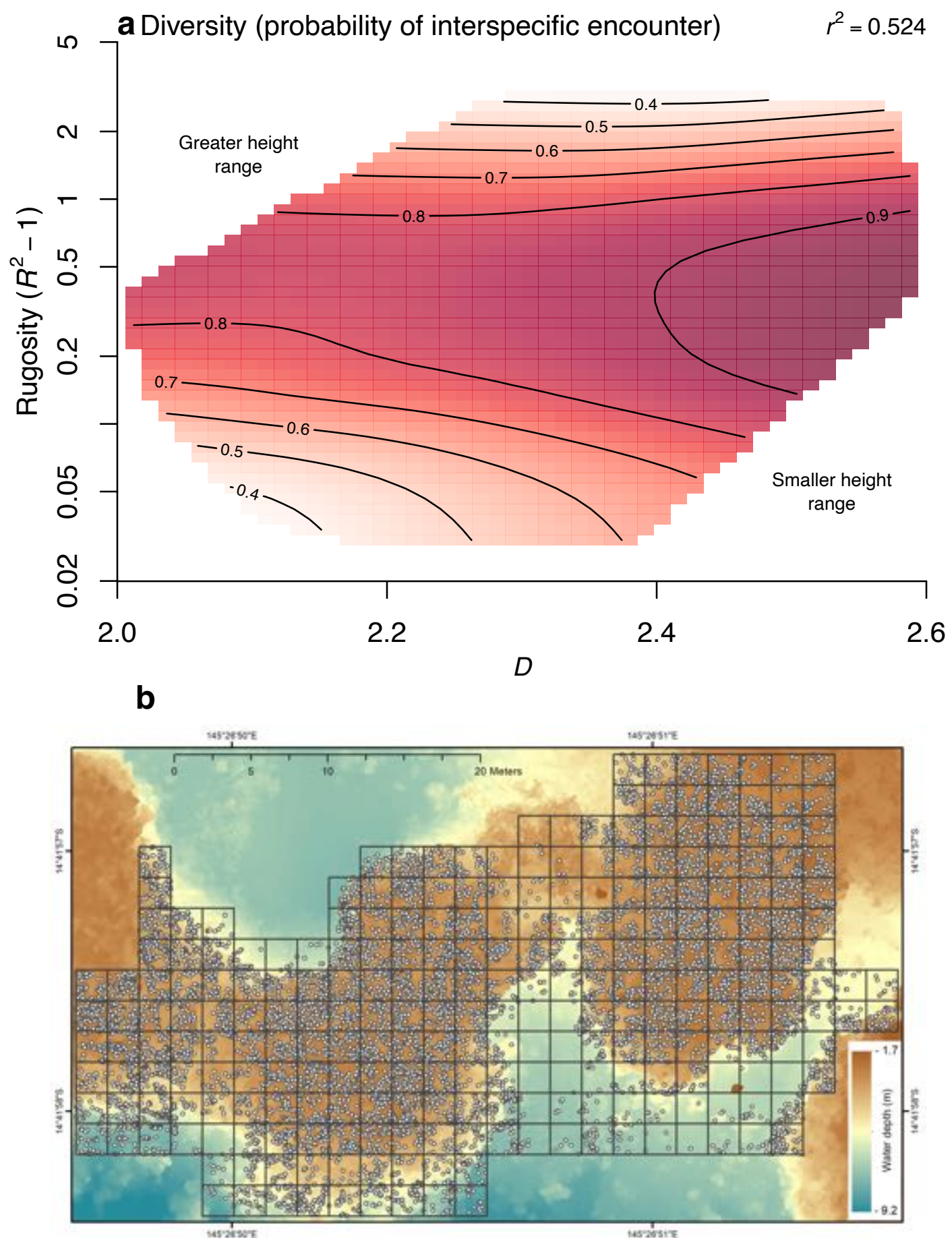